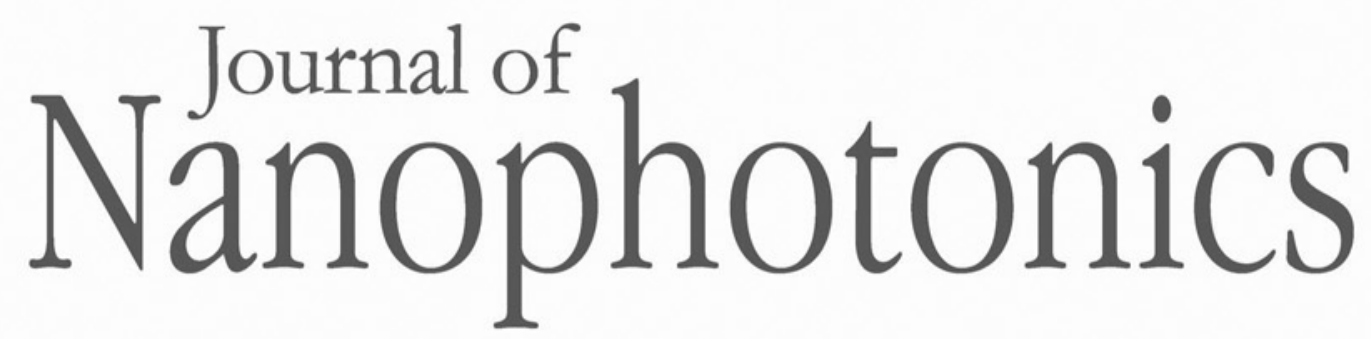

Nanophotonics.SPIEDigitalLibrary.org

\title{
Acid-free sol-gel fabrication of glass thin films embedded with II-VI colloidal quantum dots
}

Hemang Jani

Lingze Duan

\section{SPIE.}




\title{
Acid-free sol-gel fabrication of glass thin films embedded with II-VI colloidal quantum dots
}

\author{
Hemang Jani* and Lingze Duan \\ University of Alabama in Huntsville, Department of Physics, 301 Sparkman Drive, Huntsville, \\ Alabama 35899, United States
}

\begin{abstract}
II-VI colloidal quantum dots (QDs) are ideal for optical sensors thanks to their high fluorescent brightness and good size uniformity. However, embedding colloidal QDs into a glass matrix with the standard sol-gel process leads to the QDs being damaged by the acid catalyst. Here, we report an acid-free sol-gel technique, which proves to be both simple and effective in fabricating silica glass thin films embedded with commercial II-VI colloidal QDs. Octadecylamine ligands are used as a bifunctional aid to not only stabilize the QDs in solution, but also assist the formation of the $\mathrm{SiO}_{2}$ gel. We demonstrate that high-quality QD-embedded glass thin films can be developed with this technique, and our fluorescent tests indicate that, except for a small blueshift in the emission spectrum, the QDs are very well preserved through the sol-gel process. This method offers a fast and low-cost path towards thin-film QD sensors with good mechanical and thermal stabilities, which are desirable for applications involving highly focused laser beams, such as ultrafast nanophotonics. (C) The Authors. Published by SPIE under a Creative Commons Attribution 3.0 Unported License. Distribution or reproduction of this work in whole or in part requires full attribution of the original publication, including its DOI. [DOI: 10.1117/1.JNP.9.093072]
\end{abstract}

Keywords: quantum dots; sol-gels; glass; thin films; fluorescence spectroscopy; nanophotonics.

Paper 15012 received Feb. 27, 2015; accepted for publication Apr. 23, 2015; published online May 20, 2015.

\section{Introduction}

Semiconductor quantum dots (QDs) offer many unique optical properties owing to their strong spatial confinement of the charge carriers. ${ }^{1,2}$ One particular aspect that draws our research interest is the possibility of using QDs as solid-state quasi-two-level systems, whose interaction with femtosecond optical pulses can potentially lead to some interesting phenomena, such as carrierenvelop phase (CEP) sensitive population inversion. ${ }^{3,4}$ The unique features of CEP post several challenges to the QD-sensor development. First and foremost, the sensors have to be thin films of micrometer-scale thickness to prevent significant CEP slippage as the femtosecond pulses penetrating the sensors. The restricted active-layer thickness requires high QD densities in order to achieve a reasonable signal-to-noise ratio. At the same time, the QDs must be spatially dispersed enough so that they maintain their discrete, single-QD energy structures. Moreover, good size uniformity is needed for the QDs to limit inhomogeneous line broadening, and the active layer must be both thermally and mechanically stable under a tightly focused laser beam. All of these requirements can be met by using chemically synthesized II-VI colloidal QDs embedded in a transparent buffer material (matrix), such as $\mathrm{SiO}_{2}$ (glass) or $\mathrm{TiO}_{2}$, and coating the QD/matrix complex on a substrate. ${ }^{5,6}$ The sol-gel method is an effective technique to develop such QD thinfilm sensors. ${ }^{7-9}$

In this paper, we report a systematic study of sol-gel fabrication of QD-embedded glass thin films, focusing primarily on using commercial cadmium selenide (CdSe) QDs. CdSe QDs have suitable energy levels for our intended ultrafast nanophotonics research, and commercial QDs offer excellent size uniformity. Our study shows that the standard procedure of making sol-gel glass, which involves the use of acid catalysts such as $\mathrm{HCl}$ or $\mathrm{CH}_{3} \mathrm{COOH}$ (acetic acid) or $\mathrm{HNO}_{3}$

*Address all correspondence to: Hemang Jani, E-mail: hj0004@uah.edu 
(nitric acid), ${ }^{10}$ cannot be directly applied to fabricating QD-embedded glassy matrix because the acid interacts with the QDs and destroys them. We then develop an acid-free sol-gel technique, in which octadecylamine (ODA) ligands are used as a bifunctional aid in $\mathrm{CdSe} / \mathrm{SiO}_{2}$ thin-film synthesis. Our characterization indicates satisfactory film quality, and the integrity of the QDs is well preserved with the new method. Given the excellent performance and wide availability of today's commercial II-VI colloidal QDs, this technique potentially opens up a path towards simple, fast, and low-cost development of QD-glass complex systems.

\section{Sample Fabrication}

Our samples were initially fabricated using the standard sol-gel recipe ${ }^{7-9}$ which involves the hydrolysis and polycondensation of $\mathrm{Si}\left(\mathrm{OC}_{2} \mathrm{H}_{5}\right)_{4}$ (TEOS), carried out by dissolving TEOS in ethanol $\left(\mathrm{C}_{2} \mathrm{H}_{5} \mathrm{OH}\right)$ before mixing in deionized water $\left(\mathrm{H}_{2} \mathrm{O}\right)$ and hydrochloric acid $(\mathrm{HCl})$. Here ethanol serves as a mutual solvent, and hydrochloric acid is the catalyst for producing the $\mathrm{SiO}_{2}$ sol. The following mole ratio was used: TEOS: $\mathrm{H}_{2} \mathrm{O}$ : ethanol:HCL $=1: 2.47: 3.83$ : 1.82. After the glassy solution was prepared, CdSe QDs (CSE600 by NN-Labs, suspended in toluene) were added into it to form the sol. When the mixture began to show a gel-like formation, it was spin-coated on a glass substrate. The thin-film samples were tested for QD emission using a home-built fluorescence spectroscopy system as shown in Fig. 1(a). However, no fluorescence was detected. We then directly coated the QDs on the glass substrate and performed the same fluorescence test. Sharp fluorescence peaks were observed at the specified wavelength, as shown in Fig. 1(b). This leads us to believe that the QDs are damaged during the sol-gel process, in particular, by the acid catalyst.

The acid catalyst is generally needed to assist the formation of $\mathrm{SiO}_{2}$ gel. In our tests, without the acid catalyst, the TEOS glassy solution did not show any sign of gelation within several days. However, to our surprise, significant gel formation was observed when the QDs were mixed into the glassy solution $\left(\mathrm{SiO}_{2} \mathrm{sol}\right)$, even in absence of the acid catalyst. Fluorescence result further showed a clear QD emission peak, indicating that the QDs were successfully embedded into the glass. Since the commercial QDs used in these tests were suspended in a toluene solution with the help of ODA ligands, to understand which factor plays the key role in the gel formation, we added powdered QDs (with minimal ODA attached), ODA ligands, and toluene to separate glassy solutions. The solution with the ODA ligands showed a gelation process similar to the earlier observation with ODA-suspended QDs, indicating the ODA ligands can indeed assist the formation of the $\mathrm{SiO}_{2}$ gel, which is also consistent with some prior reports. ${ }^{11,12}$ To further verify this finding, we measured the gelation time of glassy solutions with different ODA concentrations. The result shows that higher ODA concentrations result in quicker gelation (see Fig. 2), indicating a direct correlation between $\mathrm{SiO}_{2}$ gel formation and the presence of ODA.

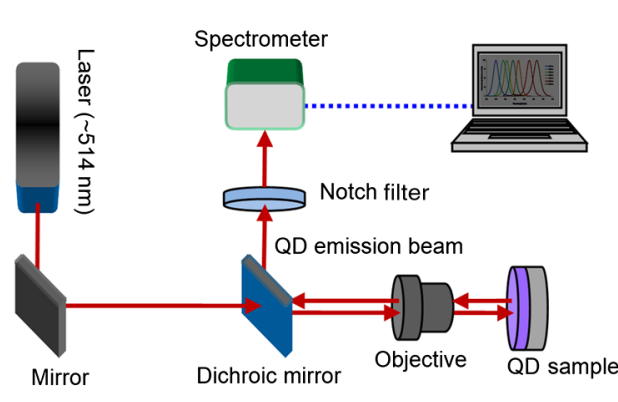

(a)

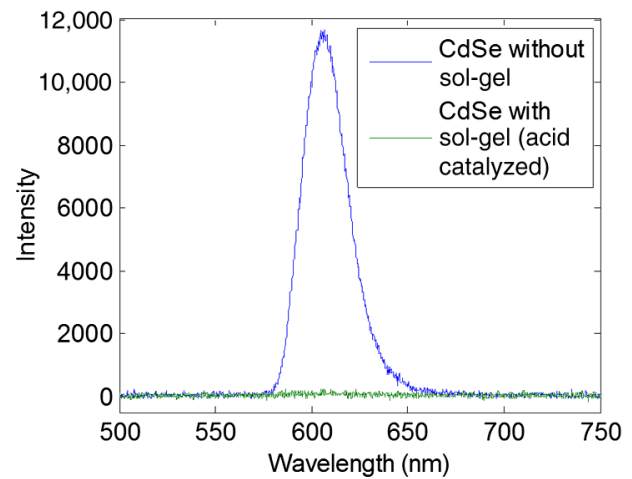

(b)

Fig. 1 (a) Schematic of the fluorescence spectroscopy setup for testing the quantum dot (QD) thin-film samples. The argon ion laser operates at a central wavelength of $514 \mathrm{~nm}$ and an output power of $12 \mathrm{~mW}$. (b) No fluorescence signal (green) was detected from the CdSe QD-embedded thin-film samples fabricated with the conventional sol-gel process using $\mathrm{HCl}$ as the catalyst, whereas strong emission signal (blue) was measured when the same CaSe QDs are directly coated on a glass substrate. 


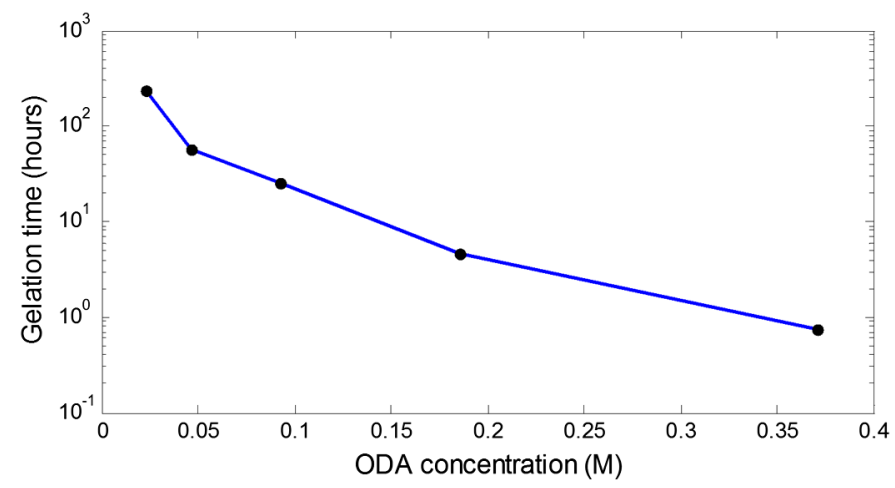

Fig. 2 The gelation time of the glassy solution versus octadecylamine (ODA) concentration indicates the effectiveness of ODA ligands in assisting the formation of the $\mathrm{SiO}_{2}$ gel.

This leads to a simple, acid-free technique to fabricate QD-embedded sol-gel glass. First, suspend the colloidal QDs in a proper solution (e.g., toluene) with the help of ODA ligands (often done by the QD vendors). Then, prepare the glassy solution out of TEOS, water, and ethanol (without adding acid). Next, mix the glassy solution and the QD solution according to the desired QD concentration to form the $\mathrm{QD} / \mathrm{SiO}_{2}$ sol. Finally, as the sol gradually turns to gel, prepare the samples based on need (e.g., coating thin films). In this scheme, the ODA ligands act as a bifunctional aid in the sol-gel process-they help stabilize the QDs in a solution and they also accelerate the gel formation.

To understand the chemistry of the acid-free technique, we note that ODA provides basic conditions for silane hydrolysis. ${ }^{11}$ Typically, base-catalyzed hydrolysis process involves an attack on silicon by a hydroxyl ion to form a pentacoordinate intermediate, followed by bimolecular displacement of alkoxy by hydroxyl. Any high-electron acceptor group next to the $\mathrm{Si}$ atom will drastically increase hydrolysis under basic $\mathrm{pH} .{ }^{13,14}$ However, even though a basic environment helps accelerate the sol-gel process, our initial attempt to switch from acid catalyst to base catalyst $\mathrm{NH}_{4} \mathrm{OH}\left(29 \% \mathrm{NH}_{3}\right)$ was unsatisfactory. It resulted in a cloudy white solution that was unsuitable for thin-film fabrication. Another study reported on ammonia quenching during sol-gel processing of QDs, which resulted in loss of luminescence over a very short period of time. ${ }^{11}$ On the other hand, our current acid-free method uses alkylamines as the sol-gel catalyst. Both ammonia and alkylamines buffer the sol-gel precursor fluid at about the same $\mathrm{pH}(\sim 9)$, but amines reduce the gelation time by two orders of magnitude. ${ }^{11}$ An additional advantage of using amines as the base catalyst is surface passivation of QDs. The surface of QDs largely determines the quantum yield and emission life-time of the bandgap luminescence (BGL). Alkylamines are excellent passivators for CdSe QDs, and they generally enhance BGL upon adsorption. Thus, the use of alkylamine yields passivated QDs and also offers a less-aggressive medium for silane polymerization.

To convert QD-embedded sol-gel glass into thin films, we spin-coated the $\mathrm{SiO}_{2}$ gel onto glass substrates at $1500 \mathrm{rpm}$ for $30 \mathrm{~s}$ and heat-treated the samples (e.g., at $\left.500^{\circ} \mathrm{C}\right)^{15}$ immediately after spin-coating. Clear, uniform films were successfully fabricated with QD molar concentrations up to $\sim 1.3 \mathrm{mM}$. Higher concentrations often yielded samples with significant cracking and much more fragile films. Currently, we are working on optimizing the postcoating procedure to improve the quality of the films at higher QD concentrations.

\section{Sample Characterization}

A picture of our thin-film sample is shown in Fig. 3(a). The thick ring-shape area around the perimeter of the coated region results from the spin-coating. The clear area in the center is the uniformly coated area. A surface profilometer trace of that area shows a uniform coating of $\sim 1 \mu \mathrm{m}$ thickness [see Fig. 3(b)]. Note that the thick ring-shape area is removed using acetone before this measurement is done. The surface quality of the film is further revealed by noncontact surface profilometry measurement. A typical result is shown in Fig. 3(c). Surface roughness in 


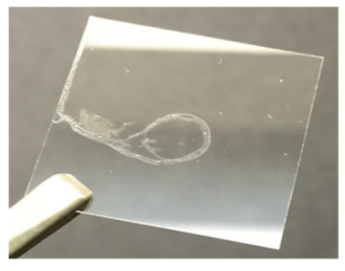

(a)

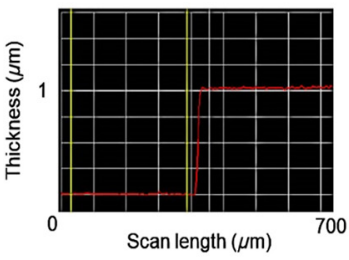

(b)

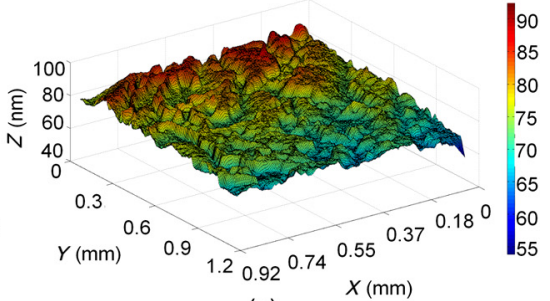

(c)

Fig. 3 (a) A spin-coated QD-embedded glass thin film on a glass substrate. (b) A measured surface profile near the edge of the film shows a smooth film with a thickness of $\sim 1 \mu \mathrm{m}$. (c) Noncontact surface profilometry data indicate that the surface roughness falls within $100 \mathrm{~nm}$ peak-to-peak over an area of $\sim 1 \mathrm{~mm}^{2}$.

general is found to fall within $100 \mathrm{~nm}$ peak-to-peak over an area of $\sim 1 \mathrm{~mm} \times 1 \mathrm{~mm}$, which indicates a very good optical quality.

More insight into the quality of the film is offered by scanning electron microscopy (SEM). Figure 4 shows the SEM images of the film in three different scales (corresponding to three different magnifications). In the largest scale (several hundred microns across), cracks can be seen on the SEM image [see Fig. 4(a)]. These cracks result from the fast drying process following sol-gel coating, which often induces mechanical stresses and hence fractures of the thin film. Nevertheless, smooth, continuous areas $>100 \times 100 \mu \mathrm{m}^{2}$ can be easily found on the film. This is sufficient to accommodate tightly focused laser beams, which typically have diameters in the vicinity of $10 \mu \mathrm{m}$. Note that the change of gray scales across the image is due to nonuniform surface-charge distribution at the time of imaging and has nothing to do with the surface profile. Meanwhile, a zoom-in view of a crack allows us to see the cross-section of the film, which indicates a fairly uniform thickness, as shown in Fig. 4(b). When the microscope view is further zoomed into a smooth area of the film, it becomes featureless, with a grainy texture [see Fig. 4(c)]. This is in agreement with the noncontact surface profilometry result shown in Fig. 3(c).

The quality of the CdSe QDs in the thin films is characterized by fluorescence measurements. In Fig. 5(a), the emission spectrum from a thin-film sample is compared with the emission spectrum from the same kind of CdSe QDs but directly spin-coated on glass substrates (i.e., without going through the sol-gel process). The similar spectral shapes of the two traces indicate that our sol-gel process did not compromise the uniformity of the QD sizes. But the emission peak from the sol-gel sample is blueshifted by $\sim 15 \mathrm{~nm}$ versus the emission peak from the unprocessed QDs (from 607 to $592 \mathrm{~nm}$ ). We believe this is caused by surface oxidization taking place during the sol-gel and coating processes. ${ }^{16}$ As oxygen permeates the outer layers of the QDs and replaces some of the Se atoms in the CdSe core, the band structure is altered and emissive exciton recombination occurs at a higher-energy transition. This effect is usually described as a reduction of the effective core size in the literature. ${ }^{16}$ To confirm this hypothesis, we performed the same sol-gel fabrication with $\mathrm{CdSe} / \mathrm{ZnS}$ core shell QDs (CZ600 by NN-Labs). The fluorescence spectrum of the core-shell sample agrees with the vendor specification, with an emission peak at $\sim 610 \mathrm{~nm}$.

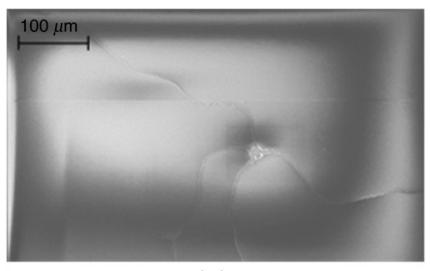

(a)

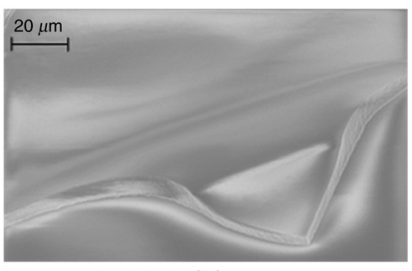

(b)

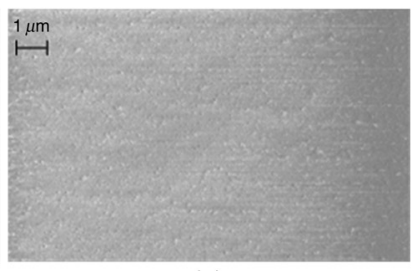

(c)

Fig. 4 Scanning electron microscopy images of the thin-film samples: (a) an overview of the central area of a thin film, (b) a close view near a crack shows the cross-section of the film, and (c) a zoom-in view of the film shows the uniform, grainy texture of the surface. 


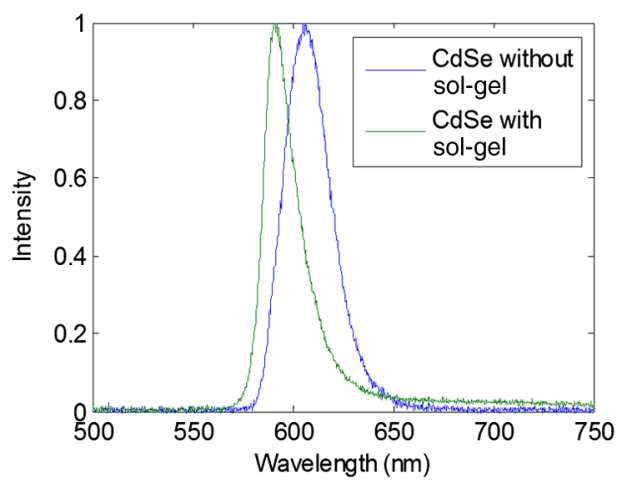

(a)

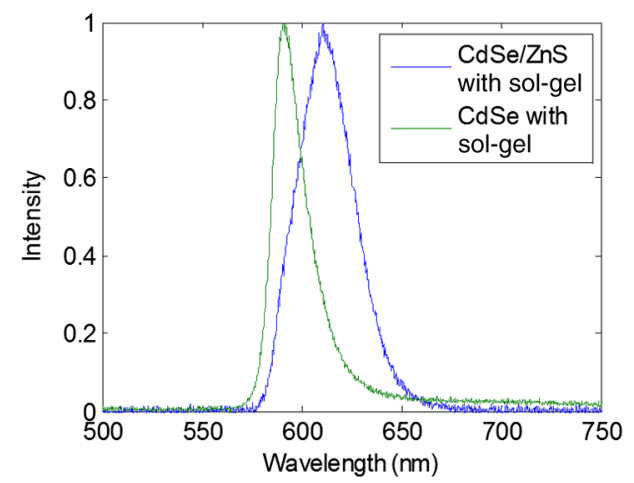

(b)

Fig. 5 (a) The emission spectrum from the CdSe QDs embedded in the glass thin film (green) shows a blueshift relative to the emission spectrum from CdSe QDs directly coated on the substrate without going through the sol-gel process (blue). (b) A similar blueshift is observed between the emission spectra of the CdSe QDs (green) and CdSe/ZnS core-shell QDs (blue), both embedded in glass thin films through the acid-free sol-gel process.

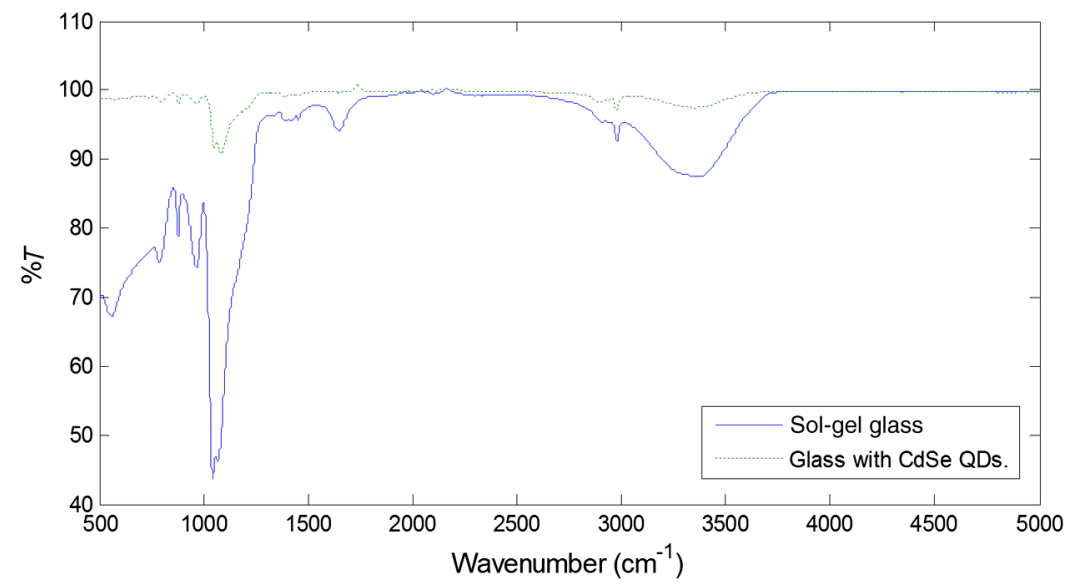

Fig. 6 The Fourier-transform infrared spectroscopy spectra of pure $\mathrm{SiO}_{2}$ glass (blue) and the $\mathrm{SiO}_{2}$ glass embedded with CdSe QDs (green).

Apparently, this can be attributed to the fact that $\mathrm{ZnS}$ shell layer prevents the CdSe core from being oxidized during the sample preparation. A comparison between the emission spectra of the CdSe sample and the CdSe/ZnS sample in Fig. 5(b) clearly illustrates the effect of surface oxidization.

To further confirm the formation of $\mathrm{SiO}_{2}$ by the acid-free technique, we performed a Fouriertransform infrared spectroscopy (FTIR) measurement with our samples, and the result is shown in Fig. 6. The blue solid trace is obtained with a glass blob produced by letting a TEOS glassy solution gradually gel up without using any catalyst. The green dashed trace corresponds to a CdSe QD-embedded thin-film sample. The two spectra show similar signature peaks near 1090 and $3430 \mathrm{~cm}^{-1}$. The former is associated with the stretching of Si-O bond, which implies that CdSe QDs are embedded in the glass matrix. ${ }^{17}$ The latter is attributed to the $\mathrm{OH}$ vibration peak from water. In addition, most of the IR spectrum peaks as mentioned in the literature ${ }^{18,19}$ are in good agreement with our results. The difference in the signal levels of the two traces is simply due to the fact that the thin-film sample has a much thinner layer to contribute to the FTIR signal.

\section{Conclusion}

In conclusion, we have developed a quick, simple, and low-cost technique to fabricate glass thin films embedded with commercial colloidal II-VI QDs. The ODA ligands are used as a 
bifunctional aid to assist both QD stabilization and gel formation. A careful characterization shows that both the film quality and the QD characteristics meet the requirements set forth by our intended ultrafast nanophotonics research. We believe this technique is a useful addition to the family of sol-gel methods and can be of special interest to researchers with limited experience or facility in sol-gel chemistry.

\section{Acknowledgments}

This research is funded in part by the National Science Foundation under grant ECCS-1254902. We thank the UAH Nano Micro Device Center, especially Frank Berisford, for providing clean room access and technical support for the thin-film development. We gratefully acknowledge Emanuel Waddell for granting us the access to Fourier-transform infrared spectroscopy and for proofreading the manuscript. We also acknowledge the contributions of Shawn R. Hays, Josiah Mullins, and Thomas Pigott in developing our understanding of sol-gel chemistry.

\section{References}

1. N. F. Borelli et al., "Quantum confinement effects of semiconducting microcrystallites in glass,” J. Appl. Phys. 61, 5399-5409 (1987).

2. B. G. Potter and J. H. Simmons, "Quantum size effects in optical properties of CdS-glass composites," Phys. Rev. B 37, 10838-10845 (1988).

3. C. Jirauschek et al., "Carrier-envelope phase sensitive inversion in two level systems," J. Opt. Soc. Am. B 22, 2065-2075 (2005).

4. C. Jirauschek et al., "Semiconductor-based carrier-envelope phase detection," presented at Conf. on Lasers and Electro-Optics (CLEO), 16-21 May 2004, Optical Society of America, Washington, DC (2004).

5. C. Li and N. Murase, "Synthesis of highly luminescent glasses incorporating CdTe nanocrystals through sol-gel processing," Langmuir 20(1), 1-4 (2004).

6. V. C. Sundar, H.-J. Eisler, and M. G. Bawendi, "Room temperature, tunable gain media from novel II-IV nanocrystal-titania composite matrices," Adv. Mater. 14(10), 739-743 (2002).

7. L. C. Klein, "Sol-gel optical materials," Annu. Rev. Mater. Sci. 23, 437-452 (1993).

8. R. M. Almeida, "Sol-gel silica films on silicon substrates," Int. J. Optoelectron. 9, 135-142 (1994).

9. J. D. Mackenzie and E. P. Bescher, "Chemical routes in the synthesis of nanomaterials using the sol-gel process," Acc. Chem. Res. 40(9), 810-818 (2007).

10. M. A. Fardad, "Catalysts and the structure of $\mathrm{SiO}_{2}$ sol-gel films," J. Mater. Sci. 35(7), 18351841 (2000).

11. S. T. Selvan et al., "Synthesis of tunable, highly luminescent QD-glasses through sol-gel processing," Adv. Mater. 13(12-13), 985-988 (2001).

12. J. Weaver et al., "Synthesis and characterization of quantum-dot polymer composites," J. Mater. Chem. 19(20), 3198-3206 (2009).

13. R. K. Nagarale, W. Shin, and P. K. Singh, "Progress in ionic organic-inorganic composite membranes for fuel cell applications," Polym. Chem. 1, 388-408 (2010).

14. Th. Materne, F. de Buyl, and G. Wittucki, "Organosilane technology in coating applications: review and perspectives," Dow Corning Review (2006).

15. X. M. Du and R. M. Almeida, "Effects of thermal treatment on the structure and properties of $\mathrm{SiO}_{2}-\mathrm{TiO}_{2}$ gel films on silicon substrates," J. Sol-Gel Sci. Technol. 8, 377-380 (1997).

16. S. R. Cordero et al., "Photo-activated luminescence of CdSe quantum dot monolayers," J. Phys. Chem. B 104(51), 12137-12142 (2000).

17. B. Sadeghimakki and S. Sivoththaman, "Investigation of structural and optical properties of CdSe quantum dots in glass matrices for photovoltaic application," Proc. SPIE 7047, 70470W (2008).

18. H. Aguirar et al., "Structural study of sol-gel silicate glasses by IR and Raman spectroscopies," J. Non Cryst. Solids 355, 475-480 (2009).

19. R. M. Almeida and C. G. Pantano, "Structural investigation of silica gel films by infrared spectroscopy,” J. Appl. Phys. 68, 4225-4232 (1990). 
Hemang Jani is a doctoral student in the optical science and engineering (OSE) program at the University of Alabama in Huntsville (UAH). His research interest is ultrafast nanophotonics. $\mathrm{He}$ received his BSc degree in physics from Maharaja Sayajirao University of Baroda, India, in 2008, his MSc degree in nuclear physics from the same university in 2010, and his MS degree in physics from UAH in 2013.

Lingze Duan received his BS degree in physics from Tsinghua University, Beijing, China, in 1995 and his $\mathrm{PhD}$ degree in electrical engineering from the University of Maryland, College Park, Maryland, in 2002. He is currently an associate professor of physics at UAH. His research interest includes ultrafast nanophotonics, frequency metrology with femtosecond frequency combs, fiber optic sensors, and novel applications of optics in astrophysics. 